\title{
30. Asymmetry of the Human Autonomic Nervous System under Stimulation of the Head by Heat
}

\author{
By Tadanobu Tsunoda \\ Medical Research Institute, Tokyo Medical and Dental University \\ (Communicated by Yasuji Katsuki, M. J. A., May 12, 1982)
}

We previously discovered difference between Japanese and nonJapanese in the laterality of brain responses to a steady-state vowel, emotional, and natural sounds, employing a key-tapping method to test cerebral hemisphere dominance. We found that the left ear advantage (LEA) for the $1 \mathrm{kHz}$ pure tone changed to the right ear advantage (REA) under emotional stimulations such as olfactory, sex and autonomic nervous system arousals. We demonstrated that this change did not take place among non-Japanese who have the central emotion faculty in the hemisphere not linguistically dominant whereas Japanese have the faculty in the linguistically dominant side. We proved, however, that the difference between Japanese and non-Japanese in the laterality of brain responses to stimuli associated with emotion and the autonomic nervous system was not due to racial hereditary factors but due to the linguistic environment in which one was raised in the first eight years or so of his life.

In the present study employing the same key-tapping test, we gave heat stimulation on many points of the left and right sides of the head alternately and found that points located over the auditory cortex and the posterior speech area in the linguistically dominant hemisphere were more deeply related to the autonomic nervous system than other points on the head.

Materials and methods. Eight Japanese subjects who were well trained to accurately perform the key-tapping test were employed. In the control test, six of the subjects showed the regular laterality (left hemisphere dominant for linguistic stimuli) while the remaining two subjects displayed the reversed laterality (right hemisphere dominant for linguistic stimuli). Specifically the six regular laterality subjects showed a REA response to a steady-vowel sound and a LEA response to the $1 \mathrm{kHz}$ pure tone. Reversed responses were observed in the two reversed laterality subjects. In addition, one Chinese subject with reversed laterality was examined and found to show a REA response to both the vowel sound and the pure tone.

In the next test using heat stimulation, the same procedure was 
repeated under administration of a hot stimulus $\left(42^{\circ} \mathrm{C}\right)$ and a cool stimulus $\left(10^{\circ} \mathrm{C}\right)$ to various points on the head. It was found that the six regular laterality subjects, this time, showed a REA response to the pure tone when a hot stimulus was given to the left side of the head or the linguistically dominant hemisphere. Then hot stimuli of $42^{\circ} \mathrm{C}$ were applied to the points on the head specified by the International Electroencephalography Society for three minutes, immediately after which responses to pure tones $(125 \mathrm{~Hz}, 250 \mathrm{~Hz}$, $500 \mathrm{~Hz}, 1 \mathrm{kHz}, 1.5 \mathrm{kHz}, 2 \mathrm{kHz}, 4 \mathrm{kHz}$ and $8 \mathrm{kHz}$ ) were measured.

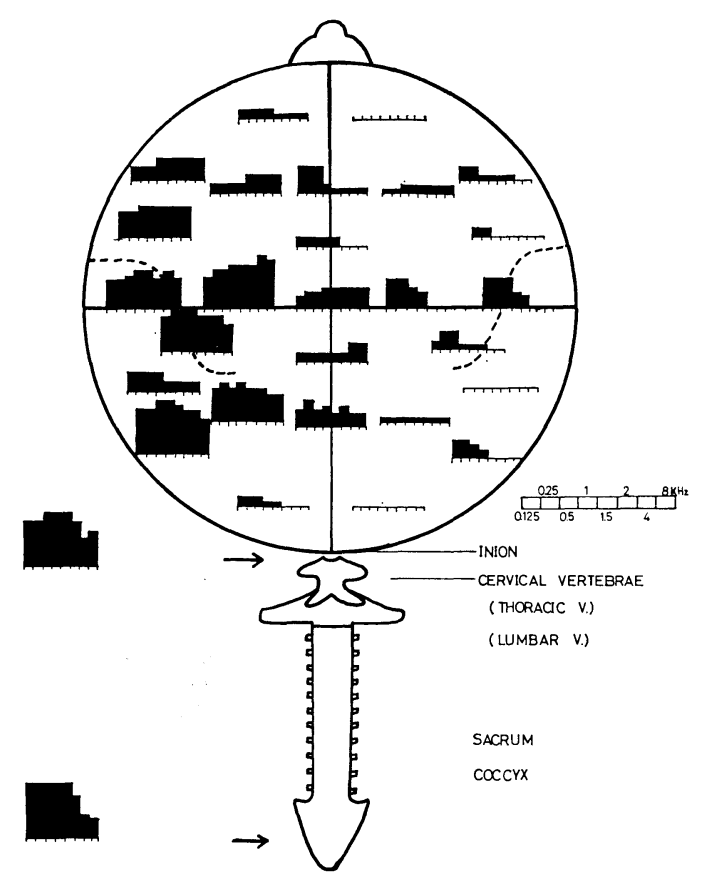

Fig. 1. Points on the head and the autonomic nervous system of Japanese, where local stimulation by heat causes the dominant hemisphere side to reverse. Frequency of the pure tone at which the dominant hemisphere side reverses under stimulation by heat was measured for six Japanese who show the regular dominant hemisphere side. The sum of the frequencies for the six is shown by the vertical coodinate.

Results and discussion. As shown in Fig. 1, the reversal of laterality from LEA to REA for the pure tone occurred most markedly in the six regular laterality subjects when hot stimulation was given to the points in the posterior and superior parts of the left half of the head corresponding to the posterior speech area and the auditory area in the brain. Though much less remarkable, 
reversal was also observed in the frontal and apex parts of the left half of the head.

The pattern of laterality reversal for pure tones indicated a tonotopic organization; that is, reversal occurred for pure tones as high as $8 \mathrm{kHz}$ in frequency under heat stimulation on the left auditory cortex area and the adjacent posterior and superior areas, whereas reversal occurred for only low frequency pure tones under heat stimulation on the frontal and peripheral parts of the left half of the head. Laterality reversal under heat stimulation in the right half of the head was minimum in area and limited to low frequency pure tones. When a steady-state vowel sound and syllable /ta/ were tested under the same hot stimulation, REA remained unaltered.

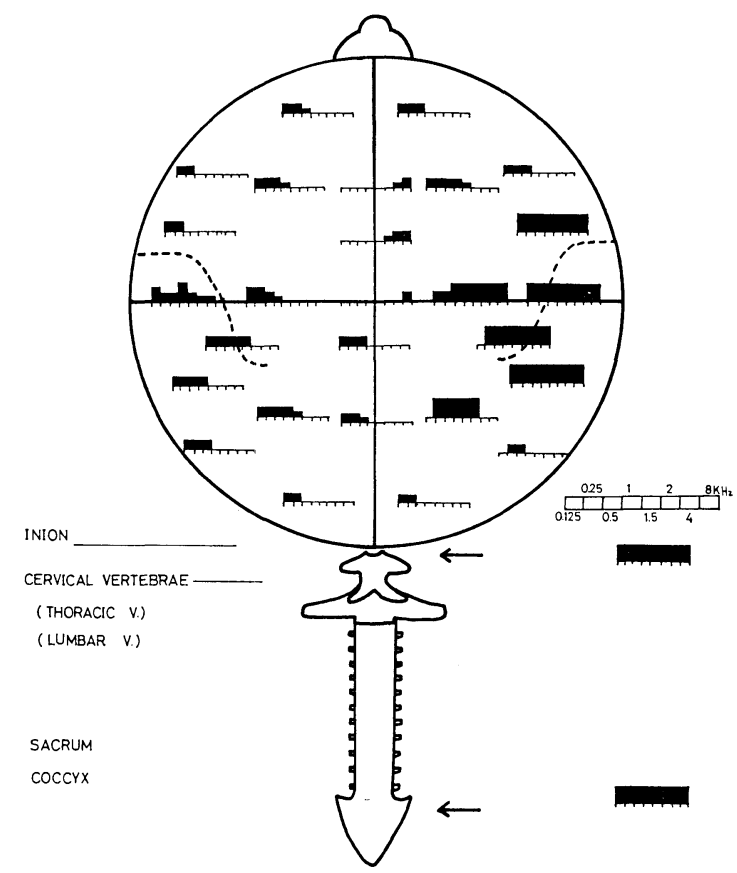

Fig. 2. Points on the head and autonomic nervous system of Japanese, where local stimulation by heat causes the dominant hemisphere side to reverse. The sum of the frequencies for the two reversed normal subjects is shown by the vertical coordinate.

As illustrated in Fig. 2, laterality reversal for pure tones was most marked in the two reversed laterality subjects when heat stimulation was delivered to the auditory cortex and speech area of the right half of the head. The reversal was rare when the left half was stimulated by hot stimulation.

Both regular laterality subjects and reversed laterality subjects, 
however, failed to show reversal under cool stimulation. It was, therefore, concluded that laterality reversal in Japanese can be induced by heat stimulation on the linguistically dominant hemisphere. The Chinese subject, on the other hand, did not show reversal responses by either hot or cool stimulation.

The difference between the Japanese and Chinese subjects in laterality reversal suggests that the linguistic function of the brain was affected by the hot stimulation via autonomic nervous system, rather than directly through the speech cortex.

To demonstrate this hypothesis, heat stimuli were applied to the points immediately below the inion, on the sacrum and on the coccyx. It was found that the dominant side for pure tones of all frequency range shifted to the linguistically dominant side in the Japanese subjects, but not in the Chinese subject (Figs. 1, 2).

Conclusion. The dominant cerebral hemisphere for the pure tone changes side in Japanese when many points on the linguistically dominant side of the head are stimulated by heat. Since similar reversal is observable in Japanese by the heat stimulation of points close to the cranio-sacral localization of parasympathetic nerves, it is believed that stimulation of the autonomic nervous system is responsible for such reversal. Western studies have found that the cerebral emotion faculty is localized in the non-linguistic hemisphere in Western people. We have confirmed that it is the case for Western subjects, but not for Japanese people who reveal the existence of the emotion faculty in the linguistic hemisphere. The fact that the Chinese subject showed different responses suggest that Chinese people, like Western people, have the emotion faculty in the nonlinguistic hemisphere which is unaffected by the autonomic nervous system. The finding of cerebral laterality reversal by stimulation of peripheral sacral areas suggests the existence of a switch mechanism for cerebral hemisphere dominance in the brain stem.

\section{References}

1) Tsunoda, T.: The characteristic pattern of the cerebral dominance for vowel sound found in Japanese second-generations. Proc. Japan Acad., 49, 643-647 (1978).

2) - : The difference of recognition mechanism toward natural sounds between Japanese and Westerners, and the spritual characteristics. Medicine and Biology, 88, 309-314 (1974).

3) : Logos and pathos: Difference in the mechanism of vowel sound and natural sound perception in Japanese and Westerners, and in regard to mental structure. Journal of Dental Health, vol. 28, pp. 35-43 (1978).

4) - : Japanese Brain. Taishukan, Tokyo (1978).

5) Tsunoda, T., and Oka, T.: Lateralization for emotion in the human brain and auditory cerebral dominance. Proc. Japan Acad., 52, 528-531 (1976).

6) Hirshkowitz, M., Karacan, I., Ware, J. C., and McCoy, G. F.: Nocturnal penile tumescence and cerebral lateralization. Oral presentation at the Third International Congress of Sleep Researches. July 27-31, 1979. 